\title{
EchoGéo
}

$33 \mid 2015$

Activisme, participation, contestation : la place des habitants dans les processus de patrimonialisation en périphéries urbaines

\section{Grands ensembles, cités ouvrières, logement social : patrimoines habités, patrimoines contestés Introduction}

\section{Elizabeth Auclair and Anne Hertzog}

\section{(2) OpenEdition}

1 Journals

\section{Electronic version}

URL: https://journals.openedition.org/echogeo/14360

DOI: $10.4000 /$ echogeo.14360

ISSN: 1963-1197

\section{Publisher}

Pôle de recherche pour l'organisation et la diffusion de l'information géographique (CNRS UMR 8586)

\section{Electronic reference}

Elizabeth Auclair and Anne Hertzog, "Grands ensembles, cités ouvrières, logement social : patrimoines habités, patrimoines contestés", EchoGéo [Online], 33 | 2015, Online since 30 September 2015, connection on 10 August 2021. URL: http://journals.openedition.org/echogeo/14360 ; DOI: https:// doi.org/10.4000/echogeo.14360

This text was automatically generated on 10 August 2021.

EchoGéo est mis à disposition selon les termes de la licence Creative Commons Attribution - Pas d'Utilisation Commerciale - Pas de Modification 4.0 International (CC BY-NC-ND) 


\section{Grands ensembles, cités ouvrières, logement social : patrimoines habités, patrimoines contestés}

Introduction

Elizabeth Auclair and Anne Hertzog

\section{Introduction}

1 Métropolisation, désindustrialisation, étalement, rénovation, gentrification, paupérisation, mise en tourisme : l'ensemble de ces dynamiques urbaines et sociales qui caractérisent banlieues et périphéries des villes françaises interagissent avec les processus de patrimonialisation, à la fois comme facteurs et comme effets, faisant de la «fabrique patrimoniale» l'une des dimensions importantes du changement urbain contemporain. Le dossier composé de 5 contributions aborde la question des processus de patrimonialisation avec une double approche, celle relative aux objets patrimoniaux qui se construisent dans ces territoires en recomposition, et celle relative aux acteurs, en questionnant les modalités de gestion collective des espaces urbains, et plus spécifiquement la place des habitants au sein de ces processus.

\section{La patrimonialisation de l'habitat populaire, question vive}

2 Les études qui constituent ce dossier sont menées à partir de terrains variés, à la fois situés en banlieue parisienne avec le cas de Plaine Commune, communauté d'agglomération de Seine Saint-Denis (et particulièrement les communes de Saint Denis, Stains et Pierrefitte) analysé par Sébastien Jacquot et Géraldine Djament-Tran, et en périphérie lyonnaise avec le cas de la Cité Tony Garnier examiné par Alain Chenevez. Les quartiers sud-est de Saint-Étienne sont analysés par Rachid Kaddour qui s'attache plus précisément au devenir des grands ensembles de Beaulieu et 
Montchovet. À travers le cas de la Cité de l'Arsenal, Diego Miralles Buil, questionne la patrimonialisation d'une cité ouvrière à la périphérie de Roanne, ville industrielle moyenne située dans le département de la Loire. En dépit de leur situation urbaine différente, les territoires analysés présentent des similarités : fort passé industriel et ouvrier, mutations liées aux effets de la désindustrialisation et aux politiques d'aménagement (rénovation urbaine), transformations sociales (gentrification ou paupérisation) qui rappellent les recompositions de nombreux quartiers périphériques des villes françaises. "Cités-jardin ", "Cités ouvrières » ou "grands ensembles ", ces territoires ont désormais intégré la longue liste des « objets patrimoniaux » urbains, à l'issue de processus de patrimonialisation récents, complexes et souvent conflictuels, engageant de nombreux acteurs.

3 Les auteurs du dossier apportent une contribution aux réflexions émergentes dans le champ des études consacrées aux processus de patrimonialisation (Auclair, Fairclough 2015 ; Gravari-Barbas, ARP, 2015) - processus à la fois culturels, sociaux, économiques et politiques - en interrogeant d'une part les effets socio-spatiaux de la "fabrique » contemporaine des patrimoines; d'autre part, le rôle de l'espace et ses dynamiques dans le rapport des sociétés urbaines contemporaines aux héritages et aux mémoires comme reconstruction du passé (Veschambre, 2007; Hertzog, 2011). Ce dossier vise à inscrire la question de la patrimonialisation dans une réflexion sur l'habiter urbain (Paquot, Younes, 2007) et l'appropriation collective et inégalitaire (Lussault, 2007, 2009) des villes. Il analyse plus précisément le rôle des habitants dans les processus de patrimonialisation, question assez largement explorée au sujet des centres anciens (Melé, 2004 ; Gravari-Barbas, 2004 ; Backouche, 2013), mais plus récente s'agissant des banlieues et des espaces périphériques urbains, où la patrimonialisation des territoires est plus tardive et se pose de surcroit en des termes nouveaux. Depuis le début des années 2000 , le contexte est en effet marqué par la montée des injonctions à la "participation habitante» dans le cadre des politiques d'aménagement urbain (politique de la Ville, rénovation urbaine, politiques de développement durable) ou des politiques patrimoniales, comme le suggère la plupart des nouveaux cadres normatifs internationaux (Déclarations et Conventions de l'Unesco ou du Conseil de l'Europe). Les contributions de ce dossier y font largement écho.

4 L'appel à textes de ce dossier proposait une approche large de la "périphérie urbaine ", de l' " habitant» et de la notion de patrimoine. Le dossier s'est néanmoins très largement recentré sur le cas des grands ensembles et des quartiers populaires des villes françaises, thématiques notablement privilégiées par les répondants à l'appel, ce qui traduit d'abord l'extension chronologique, catégorielle et spatiale de la notion de patrimoine depuis les années 1970 (Choay, 1970 ; Heinich, 2009). Ensuite, "quartier populaire ", " cité ouvrière » ou " logement social » désignent des catégories d'espaces "habités", évidemment propices à une réflexion sur le rôle des habitants dans les processus de patrimonialisation, qui était l'angle d'approche proposé par l'appel. Enfin, au-delà de l'idée que ces espaces focalisent un certain nombre des enjeux qui caractérisent les «banlieues » françaises contemporaines et leurs habitants (Desponds, Bergel, 2015), cet intérêt pour la patrimonialisation de l'habitat populaire de banlieue s'articule à une question devenue particulièrement sensible dans la recherche académique et le débat public depuis une quinzaine d'années: le devenir des grands ensembles et de leurs habitants (Dufaux, Fourcaut, 2004 ; Pouvreau, 2011 ; Veschambre, 2008). Le dossier ne prétend donc pas livrer une approche exhaustive de la diversité des 
territoires de banlieues et des types de patrimoines mais privilégie des cas de territoires périphériques touchés par les crises, et où les modalités d'engagement citoyen et de mobilisation collective demeurent très inégales (Deboulet, 2010; Deboulet, Lelévrier, 2014 ; Desponds et al., 2014).

\section{Les banlieues, des territoires au cœur des mutations urbaines contemporaines}

5 Le terme de banlieue est polysémique, et la définition première (espace situé en dehors des murs de la ville) ne suffit pas pour en révéler toute la complexité. C'est pourquoi nombre de chercheurs ont exploré cette notion pour tenter d'en donner des définitions plus précises (Merlin, 2002; Vieillard-Baron, 2001). Même si ce terme recouvre une pluralité de situations - il existe certes des banlieues aisées - il est utilisé dans ce dossier pour désigner les territoires urbains en difficulté, marqués par des problèmes économiques et une fragilité sociale (Donzelot, 2006; Stébé, 2010). D'autres notions se référant à un vocabulaire de l'aménagement ou des politiques de la Ville ont été successivement employées: ZUP (zones à urbaniser en priorité), quartiers d'habitat social, ZUS (zones urbaines sensibles), quartiers en difficulté, cités, voire même ghettos $^{1}$. La nouvelle loi sur la Politique de la ville utilise désormais la notion de «territoires prioritaires" pour désigner les quartiers faisant l'objet de dispositifs spécifiques².

Plus généralement, les banlieues sont affectées par des bouleversements qui se caractérisent à la fois par des phénomènes de densification (opérations de rénovation urbaine, réaménagement de friches industrielles, grands projets structurants...) et d'étalement (construction de nouveaux quartiers d'habitat social, de lotissements pavillonnaires et de zones d'activités), et par la construction de grandes infrastructures aggravant la fragmentation du territoire (Zembri, 2005). Sur le plan économique et social, les processus continus de désindustrialisation modifient profondément les activités et les équilibres socioéconomiques, conduisant à un accroissement massif du chômage dans certains espaces et de nombreux espaces "vacants ». Ces recompositions se traduisent par une différentiation très sensible selon les communes : dans certains cas, les territoires se caractérisent par l'installation de populations d'origine étrangère, une paupérisation croissante et une stigmatisation grandissante des quartiers, tandis que d'autres communes connaissent à l'inverse des processus de gentrification, avec l'arrivée de nouvelles catégories sociales plus favorisées, repoussant plus loin en périphérie les catégories sociales les plus modestes (Authier, Bidou-Zahariansen, 2008 ; Collet, 2015).

\section{Les processus de patrimonialisation : complexes, multidimensionnels, conflictuels}

Dans l'ensemble des contributions, la patrimonialisation est analysée comme un processus complexe, multidimensionnel et systémique, engageant de nombreuses catégories d'acteurs dotés d'intentionnalités propres et souvent divergentes. Sur le plan conceptuel, les auteurs privilégient plusieurs types d'approches: Géraldine Djament-Tran et Rachid Kaddour replacent leurs études dans les perspectives ouvertes par la géographie sociale et critique du patrimoine, insistant sur les jeux d'acteurs et 
les rapports de domination, qui caractérisent les processus de patrimonialisation dans la veine des travaux initiés à partir de la fin des années 1990 par Vincent Veschambre. Ces approches influencent également la réflexion de Diego Miralles Buil qui ancre sa contribution sur le lien patrimoine-territoire, patrimonialisation-territorialisation, fondée sur la notion fondamentale d'appropriation de l'espace (Di Méo, 1994). Cette contribution associe le processus de patrimonialisation de la Cité ouvrière à un processus complexe de reterritorialisation de l'espace urbain, suite à sa territorialisation (la conception de la Cité ouvrière en réponse à un projet économique d'État, l'Arsenal) puis à sa déterritorialisation liée au désengagement de l'État et à la "privatisation » du marché de l'immobilier, entrainant des mutations sociales). Aussi la patrimonialisation est-elle vue comme un nouveau "régime » de territorialité, contribuant à accentuer des inégalités et des contrastes hérités du passé, en instituant, selon l'auteur, une modalité de "valorisation» des anciennes cités ouvrières allant à l'encontre de leur "normalisation », entendue comme l'effacement de leur spécificité paysagère et sociale dans la ville.

8 Alain Chenevez développe une analyse qui permet de « rendre compte des prétentions à la justice patrimoniale de certains acteurs ». Revendiquant une approche inspirée des « économies de la grandeur » de Luc Boltanski et Laurent Thévenot, et transposant à la «fabrique patrimoniale » l'analyse du processus de création de l'œuvre d'art proposée par Becker, l'auteur montre que le patrimoine comme «travail interprétatif et argumentatif s'apparente à la fabrication d'une cause» par un collectif. Celui-ci implique l'ensemble d'une configuration « d'agents et d'acteurs se manifestant par des microstratégies, des alliances improbables, des interactions et des controverses, des luttes et des conflits, mais surtout un consensus minimum dans le but à atteindre ».

Pour sa part, Sébastien Jacquot aborde la réflexion sur la place des habitants dans les processus de patrimonialisation dans la perspective des travaux de Daniel Cefai sur les mouvements sociaux cherchant à " articuler des lieux et des moments, des personnes et des artefacts ». Perspective qu'il rapproche des travaux de Jacques Rancière, «articulant politique, constitution des collectifs et "constitution symbolique du social » ». L'usage de la notion de "partage du sensible », qui désigne "ce système d'évidences sensibles qui donne à voir en même temps l'existence d'un commun et les découpages qui y définissent les places et les parts respectives " s'avère alors particulièrement stimulant (Rancière, 2000 cité par Jacquot dans ce dossier). Ces apports conduisent l'auteur à proposer une approche des politiques patrimoniales à partir de la question de « ce qui fait collectif » et sur leur rôle dans la configuration des « formes de l'expérience, en mettant l'accent sur la façon dont y est défini l'habitant, sa place et ses capacités d'agir ». En ce sens, il se situe dans une perspective qui n'est pas si éloignée de celle développée par Alain Chenevez.

10 Tous les auteurs abordent la question des usages conflictuels des patrimoines et des conflits liés à la patrimonialisation des espaces urbains. Le dossier les réinterroge dans le contexte des banlieues et périphéries urbaines, en mettant en lumière les différentes modalités d'appréhension des patrimoines, ainsi que les oppositions et les tensions liés à la diversité sociale grandissante de ces espaces. Il s'agit donc ici de mesurer le poids et la place des différents groupes, d'examiner les objets et projets urbains qui suscitent refus ou adhésions, et de questionner les nouvelles échelles des conflits. La contribution de Géraldine Djament-Tran permet par exemple d'examiner dans quelle mesure l'extension catégorielle et territoriale de la notion même de patrimoine 
contribue à un glissement des conflits patrimoniaux des centres urbains vers les périphéries. Le dossier permet de questionner en quoi les échelles et conceptions renouvelées de l'aménagement urbain (intégration des territoires périphériques aux espaces centraux, projet du Grand Paris $\left.{ }^{3} . ..\right)$, ainsi que les modalités contemporaines de l'habiter en périphérie (mobilités grandissantes, nouvelles aspirations résidentielles, sentiment de relégation ou d'exclusion, éclatement des espaces de vie...) conduisent à de nouvelles formes d'appropriation conflictuelle de l'espace urbain et de conflits patrimoniaux. Les nouveaux enjeux de l'aménagement (politiques de rénovation urbaine, écoquartiers...) et le poids grandissant de certains acteurs économiques dans les transformations spatiales (promoteurs, grands groupes industriels s'implantant dans les zones franches ou espaces délaissés...) transforment les jeux d'acteurs. Ils ont pour résultat la fragilisation de certaines catégories d'habitants qui apparaissent de plus en plus démunis dans l'accès, la production ou la défense de «leur " patrimoine. Plusieurs articles mettent ainsi en lumière l'existence de groupes marginalisés ou exclus des processus de patrimonialisation face à l'action dominante de certaines catégories d'acteurs imposant leur vision légitime de la ville et du devenir urbain.

\section{Identité, justice, reconnaissance : (alter)- patrimonialisation}

11 Les différentes approches proposées par les auteurs s'avèrent particulièrement stimulantes pour penser les processus de patrimonialisation comme modalité de représentation et d'organisation du commun et du collectif dans la ville, et donc comme dimension importante de l'urbanité contemporaine ${ }^{4}$. Les contributions le rappellent bien, la patrimonialisation est l'expression culturelle, sociale, politique et

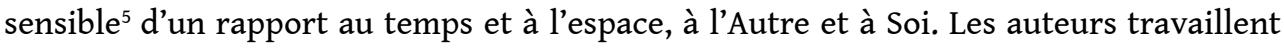
ce rapport en termes d'appropriation ou d'attachement aux lieux, mais également en termes de positionnement dans l'espace. Patrimonialiser c'est participer à la catégorisation du monde (musées), définir des limites (quartiers sauvegardés) mais aussi (se)positionner par rapport à un ailleurs ou à un autre. Dans le cas des périphéries ou des «banlieues", le positionnement - parfois militant - par rapport à un centre (dominant) est souvent l'un des enjeux des processus de patrimonialisation des territoires. Diego Miralles Buil montre que l'un des enjeux de la patrimonialisation de la cité ouvrière de Mably est de mieux relier un quartier périphérique et marginal au reste de la ville dans un souci de "cohésion urbaine " tout en affirmant une identité communale spécifique par rapport à la ville voisine de Roanne. Géraldine Djament-Tran revient sur la valorisation de la "banlieue" dans les politiques patrimoniales et touristiques de Seine-Saint-Denis, dont les enjeux sont à la fois métropolitains (repositionner des territoires périphériques) et locaux (susciter un sentiment d'appartenance, de fierté d'habiter des territoires considérés en "marge »). Sébastien Jacquot rappelle, à partir du cas de la Seine-Saint-Denis, l'émergence d'un patrimoine de banlieue: "c'est-à-dire des éléments patrimoniaux qui donnent de la consistance à la catégorie de banlieue et à son histoire, à la différence du patrimoine localisé en banlieue ». Il souligne à ce sujet que "la politique de valorisation patrimoniale devient une façon de dire une spécificité territoriale, en rupture avec la centralité parisienne: cette catégorie de "patrimoine de banlieue» n'est pas seulement un outil d'analyse, elle est revendiquée par ces nouveaux acteurs». 
12 Si l'identité de quartier de «banlieue » n'est pas explicitement revendiquée dans le cas de la patrimonialisation de la Cité Tony Garnier, l'idée d'une spécificité territoriale en rupture avec la centralité à travers la construction de nouveaux imaginaires patrimoniaux propres au grand ensemble et au quartier d'habitat social, est en revanche clairement revendiquée. Alain Chenevez montre que la patrimonialisation de la Cité Tony Garnier, engagée au départ pour la réhabilitation des immeubles dégradés, produit une " cohésion militante vécue comme fondée et juste ». Celle-ci repose sur des modalités d'action et d'énonciation revendiquant systématiquement l'écart avec les «cadres sociaux » et territoriaux conventionnels et dominants de l'art, du patrimoine et de la culture. Ainsi, la fresque murale est vue comme alternative aux formes d'expression artistiques plus légitimes, le grand ensemble est considéré comme " contre-monument» (Young, 1992), avec le soutien d'acteurs extérieurs aux institutions culturelles (Préfet du département, collectifs de peintres muraux, sociologues, agents de la politique de la Ville). En outre, l'usage de la notion de «musée » à contre-emploi pour désigner le quartier, représente un détournement de langage qui s'apparente bien à un acte de résistance, au sens où l'entend Michel Foucault, puisqu'une catégorie discursive servant à désigner le lieu de la culture légitime et élitiste par excellence est appliquée à un lieu qui n'en présente pas les caractéristiques « normées » et les critères définis par l'institution culturelle (ICOM, Ministère de la Culture, etc...). Enfin, le « collectif habitant » est perçu comme incarnation d'une forme de justice patrimoniale, à travers l'idée d'un rééquilibrage du pouvoir des experts, au profit d'une expertise « partagée » voire laissée aux seuls habitants.

Cette contribution met donc en lumière une revendication de l'écart - par rapport au centre, à la norme... - comme nouvel enjeu de légitimation, ce qui conduit à penser le processus de patrimonialisation comme une alter-patrimonialisation. Celle-ci repose sur l'invention de nouvelles modalités de définition des patrimoines, tendance qui s'affirme depuis quelques décennies, de même que leur valorisation engage de nouvelles catégories d'acteurs et des pratiques conçues comme alternatives. Patrimoine "ordinaire», "modeste», le patrimoine de banlieue renvoie aux territoires "du quotidien » et aux espaces «banals ». Ces nouveaux imaginaires patrimoniaux sont par exemple portés par la Maison de Banlieue et de l'Architecture d'Athis Mons ou encore par l'Eco-musée du Val de Bièvre à Frênes, deux structures appartenant au réseau Les Neufs de Transilie ${ }^{6}$ en région parisienne (Auclair, 2015). Ces acteurs revendiquent un questionnement sur «ce qui fait patrimoine pour les habitants» et rejettent la définition holiste et conventionnelle du patrimoine légitimant, à leurs yeux, la contestation d'un ordre socio-spatial urbain établi, et renversant les rapports de domination hérités (centre-périphérie, élite-peuple, experts-habitants, etc...). Ce ne sont plus les dimensions esthétiques, historiques ou architecturales qui sont mis en avant, mais « ce qui compte pour les habitants ", « ce à quoi ils sont attachés » (Auclair, 2014d).

14 Les contributions mettent en lumière la dimension idéologique du patrimoine et l'enjeu social et symbolique que constitue la patrimonialisation de territoires de banlieue. Elle permettrait à certains groupes sociaux « qui n'ont pas hérité de patrimoines auxquels s'identifier (châteaux, hôtels, monuments liés au pouvoir...) de se forger un patrimoine en propre, une visibilité, une légitimité », le patrimoine conférant "un droit de présence et une légitimité dans la ville » (Veschambre, 2008). 
15 En effet, dans certains quartiers périphériques fragilisés par des phénomènes de ségrégation sociale et spatiale, des ruptures à la fois physiques et symboliques s'opèrent entre les habitants et leur territoire. L'accroissement des distances entre les lieux de domicile et de travail d'une part, et entre les lieux de domicile et les structures de commerce, de culture et de loisirs, d'autre part, contribuent à ce qui apparait parfois comme une perte de repères de la part des habitants (Auclair, Vanoni, 2001). Ces processus peuvent avoir comme résultat des sentiments d'indifférence voire de rejet de la population vis à vis de leur quartier ou de leur ville. L'on assiste dans certaines villes à des processus de revalorisation dans lesquels les interventions artistiques, culturelles et patrimoniales sont appelées à jouer un rôle déterminant. De nombreux travaux ont mis en évidence la place de la culture dans les dynamiques de mise en valeur des territoires urbains (Bruston, 2005 ; Kahn, 2009 ; Ba, Zentelin, 2010), et le rôle particulier des projets culturels dans la transformation sociale des quartiers en difficulté (Chaudoir, De Maillard, 2004 ; Auclair, Brunet, 2008). Les objectifs assignés aux projets culturels et patrimoniaux sont aujourd'hui multiples : contribuer à lutter contre les phénomènes de ségrégation sociale, changer l'image des banlieues (vis à vis des personnes extérieures et mais aussi vis à vis des habitants eux-mêmes), développer un sentiment de fierté au sein de la population et renforcer un sentiment d'appartenance vis à vis du territoire, et ainsi participer à la construction d'une véritable identité territoriale (Auclair, 2003, 2006, 2010).

16 Le dossier offre cinq analyses de territoires urbains présentant des similarités sur le plan historique mais des différences sur le plan patrimonial, tant leurs «trajectoires » ou pour reprendre Alain Chenevez dans ce dossier, leurs "carrières" patrimoniales sont contrastées. Ces différences portent tout à la fois sur les processus de préservation/démolition (Beaulieu vs Montchovet à Saint-Étienne), l'inégale implication habitante (Pierrefitte vs Saint-Denis à Plaine Commune) ou encore la divergence des interprétations du passé accompagnant les pratiques de conservation/ valorisation du bâti (Office HLM vs habitants à Saint-Étienne). À l'évidence, ces « carrières patrimoniales » contrastées s'articulent à la variété des trajectoires urbaines caractérisant les périphéries des villes françaises, tout en contribuant à les accentuer. Or, les auteurs de ce dossier montrent que la patrimonialisation des territoires urbains, si elle a pu depuis des décennies être un facteur de contraste et de différenciation entre espaces centraux des villes (" sauvegardés ", « muséifiés », « touristifiés ») et territoires périphériques (Veschambre, 2007), fonctionne désormais comme un moteur de différenciation voire de fragmentation urbaine à l'échelle des périphéries elles-mêmes (Gervais-Lambony, 2007).

17 Les chercheurs mettent en lumière les logiques de valorisation/dévalorisation des territoires porteuses d'inégalités spatiales à de multiples niveaux d'échelles (Backouche et al., 2011). Les logiques de sélection et de valorisation inhérentes aux dynamiques de patrimonialisation peuvent en effet révéler de manière très crue les hiérarchisations symboliques sous-jacentes aux représentations des citadins - habitants, élus ou aménageurs...- aux registres de l'action publique ou aux projets urbains, guidant in fine, nombre de transformations urbaines. À ce titre, ce dossier illustre à travers plusieurs cas - particulièrement celui de Saint-Étienne - en quoi le processus de patrimonialisation des grands ensembles ou du logement social, peut aussi être porteur d'une « violence symbolique ", telle qu'elle a été définie par Pierre Bourdieu, à l'égard de certaines catégories d'habitants. 


\section{Entre engagement spontané et mobilisation organisée, quel rôle pour les habitants dans les processus de patrimonialisation?}

18 Au-delà des multiples travaux concernant les questions de gouvernance, des réflexions plus récentes portent sur le renouveau des théories et stratégies relatives à la participation des habitants, avec un examen des nouvelles formes de démocratie participative, et des concepts d'empowerment et de capabilités empruntés au monde anglo-saxon (Blondiaux, 2008 ; Nussbaum, 2011 ; Baqué, Biewener, 2013). Loic Blondiaux explique que l'on assiste en France comme dans la plupart des autres pays occidentaux non pas à l'émergence d'un nouveau concept, mais à la « résurgence " d'un thème qui avait été développé dans les années 60 et 70, porté à l'époque par des associations, des syndicats et des mouvements politiques (Blondiaux, 2012). Aujourd'hui, selon lui, la participation des habitants résulterait davantage d'un mouvement descendant puisque ce sont les autorités publiques élues qui en seraient les moteurs, avec une multiplication des structures ou événements de types conseils consultatifs, forums, assises...On parle d'ailleurs fréquemment, à ce propos, « d'injonction participative ».

La participation des habitants est clairement inscrite dans de nombreuses politiques publiques, et en particulier dans les politiques concernant la ville et l'environnement. En effet, depuis la Loi d'orientation pour la ville de 1991, qui définit la participation des habitants comme un principe essentiel, cette question a régulièrement été abordée par les acteurs de la Politique de la ville, même si les stratégies urbaines développées depuis 2004 dans le cadre de l'ANRU semblent avoir mis cette dimension de côté, du moins dans les premières années du programme. En effet, différents travaux ont mis en évidence le décalage entre le discours valorisant la participation des habitants, et une réalité dans laquelle les populations se retrouvent au final peu impliquées dans les projets les concernant (Desponds et ali, 2014). Suite à un rapport médiatisé soulignant la nécessité de repenser la participation des habitants (Baqué, Mechmache, 2013), la nouvelle loi de 2014 a effectivement affirmé le principe de la co-construction de la Politique de la ville avec les habitants, notamment à travers la mise en place de conseils citoyens ${ }^{7}$. Dans les domaines de l'aménagement, de l'environnement et du développement durable, on retrouve cette même importance accordée à la participation des habitants, dans de nombreux dispositifs et outils (conseils de développement pour les communautés d'agglomération et les Pays, concertation obligatoire pour les PLU, démarche participative obligatoire pour les agendas 21 locaux...). En outre, la Commission Nationale du Débat Public (devenue autorité administrative en 2002) ainsi que les Conseils de quartiers (mis en place dans les villes de plus de 80000 habitants depuis la Loi de démocratie de proximité de 2002), concourent à la structuration des dispositifs participatifs.

Concernant la participation des habitants dans le cadre des politiques culturelles et patrimoniales, la prise en compte de la population semble toujours avoir été au centre des réflexions, même si des évolutions sensibles dans les concepts et les approches ont jalonné l'histoire des politiques culturelles. On rappellera en particulier les changements liés au passage de la "démocratisation de la culture » à la "démocratie culturelle", l'objectif étant de développer les pratiques culturelles individuelles et collectives, et d'impliquer davantage les personnes non plus comme «publics » d'une 
offre culturelle, mais comme acteurs de la vie culturelle locale (Urfalino, 2004 ; Poirrier, 2010). Là encore, la participation des habitants à la vie culturelle est redevenue récemment un objet de débats, et de nombreux travaux de recherche portent sur une analyse des évolutions actuelles (Bordeaux, Liot, 2012; Gazeau, 2012). De nouveaux concepts sont mis en avant par certains acteurs et chercheurs comme celui des « droits culturels »: il ne s'agirait plus seulement de défendre un droit à la culture mais de promouvoir les droits culturels. Entre dans cette catégorie notamment le droit de prendre part de quelque manière que ce soit à la vie culturelle (Déclaration de Fribourg, 2007). Cette manière de concevoir la participation des habitants ouvre de nouvelles perspectives tout en soulevant un certain nombre de questions (Auclair, 2014c).

21 Analyser les processus de patrimonialisation sous l'angle de la «participation habitante" n'est pas qu'un effet de nouvelles préoccupations institutionnelles ou académiques. Cet angle d'attaque se justifie donc par un questionnement sur le rôle des habitants dans les périphéries urbaines, situées en dehors des quartiers historiques aujourd'hui « muséifiés » des centres-villes. Les mobilisations d'habitants, souvent issus de catégories sociales moyennes ou élevées, ont fortement contribué à l'émergence des « consciences patrimoniales» dans les quartiers centraux (Tomas, 2004; Melé, 2004 ; Veschambre in Gravari-Barbas, 2004) comme en témoignent les nombreuses associations de sauvegarde ou de défense du patrimoine des quartiers historiques (Commission du Vieux Paris créée dès 1897, Association Renaissance du Vieux Lyon créée en 1946, Sauvegarde et Avenir de Troyes en 1963...). Elles ont souvent été relayées ou coexistent avec d'autres modalités de valorisation patrimoniale de la part de nouvelles catégories d'acteurs : institutions patrimoniales protectrices, acteurs publics, acteurs touristiques... . Or, comme le montrent Alain Chenevez et Sébastien Jacquot dans ce dossier, les espaces périphériques ou de banlieue n'ont guère suscité le même type d'intérêt de la part de certaines institutions patrimoniales (services culturels et patrimoniaux de l'État, Conseils généraux, etc...) avant les décennies 1990/2000.

22 À partir d'études de terrain réalisées à Plaine Commune, privilégiant le point de vue « habitant », Géraldine Djament-Tran distingue trois figures du rapport des habitants au patrimoine qu'elle analyse comme un rapport différencié à leur espace de vie : la mobilisation de défense du patrimoine, son absence voire la mobilisation antipatrimoniale, la participation à une valorisation institutionnelle du patrimoine. La comparaison du cas de la Cité Meissonnier à Saint-Denis, de la Cité des Poète à Pierrefitte et de la Cité Jardin de Stains permet de conclure à une relativisation du rôle des habitants, que l'auteur présente d'emblée comme minoritaire. Ce trait qui serait propre aux quartiers populaires, est lié au phénomène plus global de la " désaffiliation » des catégories populaires, à la pauvreté des résidents et dans certains cas, si on suit l'auteur, à la faiblesse de l'ancrage de certains d'entre eux - ce qui pose également la question d'un patrimoine de l'immigration ou plutôt des populations d'origine immigrée habitant ces quartiers. Cependant, les «mobilisations antipatrimoniales » - s'exprimant par exemple par le désir de démolition à Pierrefitte reposent avant tout sur l'argument de la vétusté et de l'inadaptation du bâti. L'hypothèse de l'appartenance de classe, de la maîtrise d'un capital culturel supposé déterminer une « conscience patrimoniale » est également travaillée par Diego Miralles Buil au sujet du quartier de l'Arsenal, territoire caractérisé par un clivage social hérité de l'époque de la construction du quartier. L'auteur note la même indifférence à la valeur patrimoniale de la cité ouvrière, de la part des catégories populaires habitant le quartier du côté de Mably - y compris chez les anciens ouvriers, résidents de longue 
date, que de la part des néo-arrivants dans la partie roannaise de la Cité, plus huppée et en voie de gentrification. Ces populations nouvelles ignorent tout du passé du quartier et s'emploient davantage à transformer les anciennes maisons d'usine plutôt que de les restaurer ou en conserver l'authenticité. Cette démarche existe en revanche bel et bien du côté de Mably, commune populaire aux portes de Roanne, mais elle n'est pas le fait des habitants : c'est l'équipe municipale et particulièrement le Maire qui impulse la restauration de quelques maisons et surtout un aménagement de la voirie et des espaces verts respectant l'organisation urbaine ancienne dans une démarche de valorisation patrimoniale. Comme à Plaine Commune, analysée par Géraldine DjamentTran, l'auteur insiste sur la position des élus porteurs d'un «militantisme patrimonial».

\section{Actions et réactions des institutions patrimoniales : usages du patrimoine et de la patrimonialisation}

23 Le rôle déterminant des élus locaux, et notamment des Maires des communes, est évoqué par l'ensemble des auteurs, de même que celui des experts et plus particulièrement des architectes. Le dossier rappelle ainsi combien l'habitat social, les cités ouvrières ou encore les grands ensembles se sont constitués en patrimoines reconnus et labellisés par ces catégories d'acteurs, généralement extérieurs aux territoires, souvent sans les habitants, voire contre leurs revendications dans certains cas.

Alain Chenevez revient sur le cas de la Cité Tony Garnier dans l'est lyonnais, qui a fait l'objet d'un processus de patrimonialisation largement initié par certains locataires, dont quelques individus particulièrement actifs. Mais son article retrace également la véritable « reprise en main » du processus par les institutions - qu'il représente en tant que rédacteur d'un projet scientifique et culturel traduisant bien à la fois la professionnalisation et l'institutionnalisation d'un patrimoine longtemps considéré comme mineur voir illégitime aux yeux de certains acteurs culturels institutionnels. Or, à la faveur du changement des regards extérieurs, via une labellisation UNESCO et de l'émergence de nouveaux enjeux comme le développement touristique et l'aménagement métropolitain (Grand Lyon puis Lyon Métropole), cette réappropriation institutionnelle entraîne la marginalisation, voire l'éviction des habitants au sein du processus de valorisation.

Les articles permettent donc de questionner la manière dont la notion de «coproduction" patrimoniale, encouragée par plusieurs textes internationaux ${ }^{8}$ et notamment la convention de Faro (non signée par la France à ce jour), s'incarne dans les pratiques (Fairclough et al, 2014). Les articles examinent ainsi la diversité des rôles joués par les institutions vis à vis des mobilisations habitantes (aiguillon, moteur, accompagnement, frein...), et mettent en lumière les nouvelles formes de partenariat qui s'élaborent entre ces institutions et les populations, sur la question de la patrimonialisation des espaces urbains. Les articles montrent la diversité des acteurs impliqués - associations, musées, institutions culturelles et patrimoniales, collectivités territoriales, ou organismes gestionnaires - source de « polyphonie », mais plus encore de " cacophonie », comme le souligne Rachid Kaddour. patrimoniales dans le contexte de la métropolisation, des recompositions des 
périphéries (Grand Paris, rénovation urbaine, montée des écarts de richesse, formation des communautés d'agglomération...) et des nouveaux impératifs de l'aménagement urbain. La prise en compte de la diversité des publics et de l'environnement social et territorial au sein des dispositifs "participatifs ", s'inscrit dans de nouveaux impératifs comme la «durabilité ", la "diversité » et la «cohésion» dans un espace urbain souvent vu comme "fragmenté », "polycentrique » ou "éloigné ». La montée de la demande sociale et politique suscite chez les professionnels du patrimoine de nouveaux questionnements sur la place à donner aux habitants dans la définition (Delarge, 2011) et les méthodologies d'identification et d'inventaire des patrimoines urbains (Auduc, 2008, 2011; Auduc, Faure, Hertzog, Auclair, 2014). Le dossier permet de mieux saisir comment la notion d' " habitant" qui fait l'objet de réflexions renouvelées dans le champ des sciences sociales (Anquetin, Freyermuth, 2009; Tricot, 2014; Auclair, Faircough, 2015), est mobilisée dans les discours des acteurs culturels, des professionnels du patrimoine et de l'aménagement, et des élus, notamment à travers la contribution de Sébastien Jacquot. S'intéressant aux différentes «façons dont les habitants sont interpellés et catégorisés par les politiques de valorisation patrimoniale ", l'auteur distingue cinq «figures habitantes » définies à partir de la place et du rôle qui leur sont donnés dans les politiques publiques de valorisation patrimoniale touristique: bénéficiaires, témoins, touristes, ambassadeurs, participants/co-producteurs. À partir de l'analyse du Schéma touristique communautaire de Plaine Commune 2012-2016, l'auteur montre l'usage différencié de la notion d'habitant conduisant notamment à un brouillage des catégories habitants/ touristes, le recours à la catégorie de l'habitant-touriste permettant la construction d'une posture de «redécouverte» du territoire du quotidien visant à sa " réappropriation ", dont on a vu qu'elle constitue l'un des grands enjeux des politiques patrimoniales participatives contemporaines. Ainsi la désignation des « habitants » au sein des politiques publiques participative vise à définir la place attribuée à chacun dans la ville, son rôle et sa capacité à agir au sein d'un territoire.

Diego Miralles Buil montre que la patrimonialisation de la cité ouvrière à Mably est un processus pris en charge par la municipalité communiste, dont les habitants sont avant tout des destinataires. Néanmoins certains d'entre eux, les « anciens ouvriers » ont été mobilisés en tant que " témoins » (rassemblement de témoignages sur le quartier...) et porteurs d'une « mémoire ouvrière » - improbable « mémoire de classe » (Verret, 1984) dans le contexte de recomposition socio-spatiale de la ville.

Le dossier illustre aussi la récurrence de certaines pratiques participatives dans les quartiers populaires, notamment l'appel aux «mémoires habitantes» et leur patrimonialisation. Ces pratiques institutionnalisées et désormais systématisées en contexte de rénovation urbaine, suscitent depuis longtemps un certain nombre de questionnements et de critiques (Foret, 2007) notamment le risque de leur « instrumentalisation » (Auclair, 2014 a et b), que souligne également l'étude de Rachid Kaddour sur Saint-Étienne. L'auteur oppose deux types de patrimonialisation inégalement réalisées. Celle de la "mémoire du mouvement HLM » à Beaulieu, conduit à un modèle de patrimonialisation réalisée (bâti conservé, labellisé, mémoires valorisées et rendues visibles dans l'espace public) reposant sur l'ultra sélectivité mémorielle, l'occultation de pans entiers de l'histoire urbaine récente - et sur l'éviction de certaines mémoires. Celle des habitants de Montchovet, dont les témoignages et souvenirs ont été recueillis au moment de la démolition du grand ensemble en 2000. 
29 Assiste-t-on à un processus de patrimonialisation de ces mémoires, qualifiées par Rachid Kaddour de "patrimoine immatériel »? Certes, pour les habitants participants, les récits, dessins et réalisations diverses constituent les supports d'expériences importantes permettant d'exprimer autant que d'« éprouver ». C'est peut-être aussi l'occasion de transmettre collectivement le rôle de la communauté (maghrébine notamment), l'importance de la précarité et de la violence, mais aussi des luttes, dans le mode d'habiter. Mais elles renvoient pour les acteurs institutionnels à des mémoires peu légitimes car "négatives» et dissonantes (ghettoïsation, ségrégation, «contremodèle » urbain...), ce qui, de fait, les rend moins visibles et plus vulnérables. L'auteur oppose ainsi les « supports soignés et durables et réalisés avec des moyens significatifs : des films, un ouvrage et l'appartement reconstitué » fixant les mémoires des habitants de la première heure de Beaulieu, aux supports "éphémères et réalisés sans moyen : prise de parole lors de fêtes, impression de paroles sur support papier simple diffusés dans un cercle très restreint » caractérisant les mémoires de locataires plus pauvres et arrivés récemment. Ces mémoires des locataires maghrébins de Montchovet perçu comme un "ghetto » et plus encore, les récits de luttes face aux institutions pour l'équipement $d u$ quartier ou contre les démolitions peuvent être vus comme des « contre-mémoires » en référence à Michel Foucault, occultées voire "silencieuses » dans l'espace public, les empêchant d'accéder à un statut de patrimoine pour d'autres acteurs que les seuls habitants.

L'idée selon laquelle la valorisation patrimoniale, qui implique une connaissance renouvelée de territoires longtemps ignorés, dévalorisés ou stigmatisés (Beaulieu à Saint-Étienne, la Cité ouvrière à Mably, la Cité Jardin à Stains), susciterait une reconnaissance des habitants, par leur ré-intégration au " grand récit » urbain et leur mise en visibilité dans l'espace urbain, est une idée communément admise qui revient de façon récurrente dans les discours des acteurs et des chercheurs. En ce sens, la "patrimonialisation " incarne une "idéologie spatiale " urbaine au sens où l'entend Michel Lussaut (2007), guidant un certain nombre de pratiques et de politiques urbaines. Cependant, comme en témoignent ces contributions, les processus différenciés de patrimonialisation, processus de valorisation tant symboliques que matériels, participent pleinement aux «systèmes des inégalités » (Veschambre, 2007, 2008).

\section{BIBLIOGRAPHY}

Anquetin V., Freyermuth A., (dir.), 2009. La figure de l"'habitant". Sociologie politique de la demande sociale. Rennes, PUR, $184 \mathrm{p}$.

Auclair E., Fairclough G., (dir.), 2015. Theory and practice in heritage and sustainability: living between past and future. London Routlege.

Auclair E., 2015. Ordinary heritage, participation and social cohesion in the banlieues of Paris. In Auclair E., Fairclough G., (eds), Theory and practice in heritage and sustainability: living between past and future. London, Routlege. 
Auclair E., 2014a. Artistes, habitants et démolition : le rôle des projets culturels dans la rénovation urbaine. In Desponds D., Auclair E., Bergel P., Bertucchi MM., (dir), Les habitants, acteurs de la rénovation urbaine? Rennes, PUR, p. 111-126.

Auclair E., 2014b. Les projets mémoriels en banlieue : participation ou instrumentalisation des habitants ? Revue Francosphères, $n^{\circ}$ 3-2.

Auclair E., 2014c. Diversité culturelle et droits culturels, atouts et ambiguités des nouveaux concepts mobilisés dans les quartiers. In Bertucci M.M., Boyer I., Bulot T. (dir), Diasporisations sociolinguistiques et précarités. L'Harmattan, p. 145-162.

Auclair E., 2014d. Paysages ordinaires de banlieue : reconnaissance et appropriation par les habitants. Paysages urbains d'Ile de France, Paris, Somogy éditions d'art.

Auclair E., 2010. L'action culturelle peut-elle constituer un vecteur d'intégration sociale et devenir un véritable outil de valorisation du territoire? In Hameth Ba A., Zentelin J.L. (dir), La dimension culturelle du développement. Dynamiques de valorisation et de dévalorisation des territoires urbains. L'Harmattan.

Auclair E., Brunet F., 2008. Projets culturels et développement social des quartiers, retours d'expériences et perspectives. Recherche Sociale, $n^{\circ} 187$.

Auclair E., 2007. La culture et les quartiers populaires. Diversité, $n^{\circ} 148$, p. 53-59.

Auclair E., 2006. Comment les arts et la culture peuvent-ils participer à la lutte contre les phénomènes de ségrégation dans les quartiers en crise ? Hérodote, $\mathrm{n}^{\circ} 122$.

Auclair E., 2003. Le développement culturel comme outil de promotion d'une identité territoriale, ou comment les acteurs locaux se saisissent de la culture pour faire émerger un territoire. In Gravari-Barbas M., Violier P. (dir), Lieux de Culture, culture des lieux. Presses universitaires de Rennes.

Auclair E., Vanoni D., 2001. Ville émergente, le fonctionnement de la multicentralité. Recherche sociale, $n^{\circ} 157$.

Auduc A., 2008, Patrimoines d'Ile-de-France. Rencontre des histoires, des populations et des territoires. Actes du colloque régional, 6-7 décembre 2007, Région Île-de-France, Somogy Editions d'art, $208 \mathrm{p}$.

Auduc A. (dir), 2011. Ces patrimoines qui font territoire. Actes du colloque régional, 24-25 novembre.

Auduc A., Faure J., Auclair E., Hertzog A., 2014. La patrimonialisation en question. Entre culture experte et culture habitante. Projet de recherche sélectionné dans le cadre de l'appel à projets du GIS IPAPIC 2013 : « Pratiques interculturelles dans les processus de patrimonialisation », Ministère de la Culture et de la Communication. Voir Ministère de la Culture et de la Communication, 2015, Culture et Recherche, 130, p. 98-99.

Authier J.-Y., Bidou-Zahariansen C. (dir), 2008. La gentrification urbaine. Espaces et sociétés, $n^{\circ}$ 132-133.

Backouche I., Ripoll V., Tissot S., Veschambre V., 2011. La dimension spatiale des inégalités. Rennes, PUR.

Backouche I., 2013. Aménager la ville. Les centres urbains français entre conservation et rénovation (de 1943 à nos jours). Paris, A. Colin, 448 p.

Baqué M.-H., Biewener, C., 2013. L'empowerment, une pratique émancipatrice. La Découverte. 
Baqué, M.-H., Mechmache, M., 2013. Pour une réforme radicale de la Politique de la ville. Rapport remis au gouvernement.

Blanchard, P., 2013. Histoires, patrimoine et mémoires dans les territoires de la politique de la ville. Rapport remis au Ministre délégué à la ville François Lamy.

Blatrix C., 2009. La démocratie participative en représentation. Sociétés contemporaines, $\mathrm{n}^{\circ} 74$.

Blondiaux L., 2008. Le nouvel esprit de la démocratie. Actualité de la démocratie participative. Le Seuil.

Bordeaux M.-C., Liot, F. (dir), 2012. La participation des habitants à la vie artistique et culturelle. L'Observatoire, $\mathrm{n}^{\circ} 40$.

Bruston A. (dir), 2005. Des cultures et des villes, Mémoires au futur. Éditions de l'Aube.

Callon M., Lascoumes P., Barthe Y., 2001. Agir dans un monde incertain, essai sur la démocratie technique. Paris, Seuil, 358 p.

Carrel M., 2013. Faire participer les habitants, Citoyenneté et pouvoir d'agir dans les quartiers populaires. ENS Éditions.

Chaudoir P., Maillard J. (dir), 2004. Culture et politique de la ville. Éditions de l'Aube

Choay F., 1970. L'Allégorie du patrimoine. Paris, Ed du Seuil

Collet A., 2015. Rester bourgeois. Les quartiers populaires, nouveaux chantiers de la distinction. Paris: La Découverte.

Deboulet A., Lelévrier C., 2014. Rénovations urbaines en Europe. Rennes, PUR.

Deboulet A., Nez H., 2013. Savoirs citoyens et démocratie urbaine. Rennes, Presses Universitaires de Rennes.

Deboulet Agnès et al., 2010. La rénovation urbaine entre enjeux citadins et engagements citoyens. Rapport de recherche, Éditions du Puca.

Delarge A., Ecomusées du Val de Bièvre, 2011. Le patrimoine de banlieue existe-t-il ? Actes de la journée d'étude à l'écomusée du Val de Bièvre, 2 février 2010.

Desponds D., Bergel P., 2015. Les « banlieues françaises » : des dynamiques complexes derrière un mot trop ordinaire. In Carpenter J., Horvath C. (dir.), Regards croisés sur la banlieue, Peter Lang, Bruxelles. p. 23-38.

Després C., Fortin A., Vachon G., 2001. La banlieue, patrimoine ? Quelques éléments de réflexion. Québec, Patrimoine, p. 25-28.

Di Méo G., 1994. Patrimoine et territoire, une parenté conceptuelle. Espaces et société, n78, 1994/4

Djament-Tran G., 2014. La métropolisation de la culture et du patrimoine. Paris, Ed. Le Mansucrit ;

Donzelot J., 2006. Quand la ville se défait : une politique face à la crise des banlieues. Seuil.

Douay N., Prevot M., 2012. Numéro spécial « Activismes urbains ». L’information géographique, vol. $76 / 2$ et vol 76/3.

Dufaux F., Gervais-Lambony Ph, 2009. Justice spatiale. Annales de Géographie, 2009/1, n 665-666.

Dufaux F., Fourcaut A. (dir.), 2004. Le monde des grands ensembles. Paris, Créaphis, 288 p.

Fairclough G., Dragicevic Sesic M., Rogac Mijatovic L., Auclair E., Soini K., 2014. The Faro Convention, a new paradygme for socially - and culturally- sustainable heritage action? Kyлtypa/ Culture $n^{\circ} 8$ (journal for cultural researches, publié par MI-AN Publishing, Centre for Culture and Cultural Studies, Skopje, République de Macédoine). 
Fourcaut A., 2000. Pour en finir avec la banlieue. Géocarrefour, vol. 75, n 75/2, p. 101-105.

Fourcaut A., 2007. Les quartiers populaires ont aussi une histoire. Projet, 2007/4, 299, p. 7-15

Frère.B, Jacquemain M., 2013. Résister au quotidien ? Les Presses de Sciences Po.

Gazeau S (dir), 2012. Les projets participatifs au cœur de la (politique de la) ville. Art factories/ autrepArt(s).

Gravari-Barbas M. (dir), 2004. Habiter le patrimoine. PUR.

Gravari-Barbas M. (dir.), 2014. Nouveaux défis pour le patrimoine culturel. Atelier de Réflexion Prospective, Rapport final, ANR.

Groueff S., 2014. Militantisme patrimonial en banlieue. Urbanisme, 369, p. 39-41.

Hameth Ba A., Zentelin J.-L. (dir), 2010. La dimension culturelle du développement. Dynamiques de valorisation et de dévalorisation des territoires urbains. L'Harmattan.

Hamon F., 2007. Grands ensembles, demande de patrimonialisation et base Mérimée. Histoire urbaine, 2007/3 n²0, p. 125-132. http://www.metropolitiques.eu/Faut-il-patrimonialiser-lesgrands.html

Hatzfeld H., 2014. La participation, une histoire ancienne. Urbanisme, 392, p. 32-35.

Healey P., 2003. Collaborative Planning in Perspective. Planning Theory, 2/101 - SAGE.

Healey P., 1997. Collaborative planning, shaping places in fragmented societies. Vancouver, University of British Columbia Press.

Heinich N., 2009. La fabrique du patrimoine. De la cathédrale à la petite cuillère. Éditions de la Maison des Sciences de l'Homme, coll. Ethnologie de la France, $286 \mathrm{p}$.

Hertzog A., 2014. Habiter la ville en transformation. Rénovation urbaine et mobilisation habitante à Vitry sur Seine: art, mémoire et activisme urbain. In Desponds D., et al. Les habitants, acteurs de la rénovation urbaine. PUR.

Hertzog A., 2011. Les géographes et le patrimoine. EchoGéo [En ligne], 18 | 2011, mis en ligne le 05 décembre 2011. URL : http://echogeo.revues.org/12840 ; DOI : 10.4000/echogeo.12840

Lefebvre, H., 1968. Le Droit à la ville. Anthropos ( $2^{\mathrm{e}}$ ed.), Paris, Ed. du Seuil.

Lussault M., 2009. De la lutte des classes à la lutte des places. Grasset.

Lussault M., 2007. L'homme spatial, la construction sociale de l'espace humain. Seuil.

Luxembourg C., 2014. Métamorphose des villes industrielles. Vivre la ville désindustrialisée.

L'Harmattan.

Melé P., 2004. Habitants mobilisés et devenir d'un espace patrimonial ? Géocarrefour [En ligne], vol. 79/3 | 2004, mis en ligne le 12 mars 2008, consulté le 22 septembre 2015. URL : http:// geocarrefour.revues.org/734

Melé P., 2013. Conflits de proximité et dynamiques urbaines. Rennes, PUR.

Merlin P., 2002. Les banlieues. PUF.

Nussbaum, M., 2011. Capabilités, comment créer les conditions d'un monde plus juste ? Paris, Climats.

Paquot Th., Younes C. (dir.), 2007. Habiter le propre de l'humain. La Découverte.

Poirrier, P. (dir), 2010. Politiques et pratiques de la culture. La Documentation française, 2010. 
Pouvreau B., 2011. Faut-il « patrimonialiser » les grands ensembles ? Métropolitiques, 28 mars. URL : http://www.metropolitiques.eu/Faut-il-patrimonialiser-les-grands.html

Rautenberg M., 2003. La rupture patrimoniale. À la croisée, Bernin.

Ripoll F., 2008. Espaces et stratégies de résistance : répertoire d'action collective dans la France contemporaine. Espace et sociétés, $\mathrm{n}^{\circ} 134$, p. 83-97.

Stébé J.-M., 2010. La crise des banlieues, sociologie des banlieues sensibles. PUF.

Tricot A., 2014. L'habitant, un « amateur » d'espace ? Réflexions théoriques et méthodologiques pour une analyse de la perception sensible de l'environnement et du risque. In Ferrié J-N, Tournay V., Weisbein J., (dir), La consistance des êtres collectifs échanges pragmatiques et enjeux épistémologiques. Herman.

Urfalino P., 2004. L'invention de la politique culturelle. Hachette.

Verret M., 1984. Mémoire ouvrière, mémoire communiste. Revue Française de Science Politique, $\mathrm{n}^{\circ} 3$, p. $413-427$.

Veschambre V., 2000. Firminy-Vert et le "site Le Corbusier". Vers une esthétisation du grand ensemble? Cahiers de la Méditerranée, n60, 1, 2000. Paysages urbains (XVIe-XXe siècles). Tome II [Actes du colloque de Grasse, décembre 1998] p. 133-156.

Veschambre V., 2008. Traces et mémoires urbaines, enjeux sociaux de la patrimonialisation et de la démolition. Rennes, Presses universitaires de Rennes.

Vieillard Baron H., 2001. Les banlieues, des singularités françaises aux réalités mondiales. Paris, Hachette Supérieur.

Young J.-E., 1992. The Counter-Monument. Memory against Itself in Germany. Critical Inquiry, 18, p. 267-296.

Zembri, P., 2005. Les nouvelles périphéries urbaines : pour une relativisation de la notion classique de banlieue. In Bertucci M.M., Houdard Mérot, V. (dir), Situations de Banlieue, Lyon, INRP.

\section{NOTES}

1. Au lendemain des attentats de janvier 2015 à Paris, le premier Ministre Manuel Valls utilisa l'expression «apartheid territorial, social et ethnique » pour décrire la situation de certains quartiers, non sans créer des polémiques quant à l'usage du terme apartheid.

2. La Loi n 2014-173 du 21 février 2014 de programmation pour la ville et la cohésion sociale identifie 1300 territoires prioritaires.

3. Loi $n^{\circ}$ 2010-597 du 3 Juin 2010 relative au Grand Paris.

4. En France, mais aussi dans le reste du monde, tant les processus de patrimonialisation connaissent une extension mondialisée, quoiqu'inégale et différemment configurée selon les contextes.

5. Au sens où elle mobilise les émotions, les sens, le rapport à l'esthétique, la perception de l'environnement matériel et l'usage d'objets

6. Ce réseau informel de réflexion et de recherche créé en 2003 regroupe une quinzaine d'établissements culturels dont l'objectif est « de proposer un regard différent et renouvelé sur les réalités d'un territoire francilien contrasté et complexe ».

7. Loi du 21 février 2014 de programmation pour la ville et la cohésion urbaine. 
8. Ainsi les conventions du Conseil de l'Europe (Convention européenne sur le paysage de 2000, Convention de Faro de 2005 sur la valeur du patrimoine culturel pour la société) et les textes et conventions de l'Unesco (Convention sur la diversité culturelle de 2005, Recommandation de 2011 sur les paysages urbains historiques) encouragent les États à considérer le patrimoine non pas comme des ensembles d'objets à préserver mais comme des processus, donnant une place importante au patrimoine ordinaire et accordant aux habitants un rôle dans la mise en œuvre des projets et politiques patrimoniales

\section{ABSTRACTS}

Metropolitan growth, de-industrialization, urban sprawl, renovation, gentrification, impoverishment and tourism development are among the many urban and social changes that affect suburbs and peripheries of French towns and cities. These processes interact with the attribution of heritage status to some areas. The present number of this journal contains five articles that explore aspects of the recognition of urban heritage. Attention is focused on heritage features in areas undergoing recomposition, and on 'actors' involved, especially the position of residents in these processes.

\section{INDEX}

Subjects: Sur le Champ - Sur le Terrain

\section{AUTHORS}

\section{ELIZABETH AUCLAIR}

Elizabeth Auclair, elizabeth.auclair@u-cergy.fr, est Maître de conférences en aménagement à l'Université de Cergy-Pontoise, Directrice du Master Développement culturel et valorisation des patrimoines et membre du Laboratoire MRTE. Elle a publié récemment :

- Auclair E. ,2015. Ordinary heritage, participation and social cohesion in the banlieues of Paris. In Auclair E., Fairclough G, (dir), Theory and practice in heritage and sustainability: living between past and future. London, Routlege, Studies in Culture and Sustainable Development.

- Auclair E., 2014. Artistes, habitants et démolition : le rôle des projets culturels dans la rénovation urbaine. In Desponds D., Auclair E., Bergel P., Bertucchi M.-M. (dir), Les habitants, acteurs de la rénovation urbaine? Rennes, PUR.

- Auclair E., 2014. Les projets mémoriels en banlieue : participation ou instrumentalisation des habitants? Revue Francosphères, n³-2.

\section{ANNE HERTZOG}

Anne Hertzog, hertzog.anne@wanadoo.fr, est Maître de conférences en géographie à l'Université de Cergy-Pontoise et membre du laboratoire MRTE. Elle a publié récemment :

- Hertzog A., 2015. The challenge of cultural sustainability in city museums: Showing the city and selecting past in Noyon (France). In Auclair E., Fairclough G., Theory and Practice in Heritage and 
Sustainability: Between past and future. London, Routledge, Studies in Culture and Sustainable Development.

- Hertzog A., 2014. Reinventing battlefield tourism « in time of peace » : connecting tourism and violence. In Andrews Hazel, Tourism and violence. Ashgate Publishing Ldt.

- Hertzog A., 2014. Le musée d'art contemporain de banlieue, un nouveau territoire du tourisme en périphérie métropolitaine ? Le Mac/Val de Vitry sur Seine. In Gravari-Barbas M., Tourisme et Métropolisation. Une approche parisienne. Belin, coll. Mappemonde. 